\title{
COMMISSION 30: RADIAL VELOCITIES (VITESSES RADIALES)
}

\author{
Report of Meeting, 19 August 1970
}

President: D. S. Evans.

SeCretary: A. H. Batten.

The President in the chair.

Administrative:

Dr H. A. Abt, currently Vice-President, desired by letter to withdraw from office because of other heavy commitments. The Commission endorsed the proposed names of new officers.

No objection was raised to the text of the printed Commission report.

Scientific Communications:

Beardsley described observations of standard velocity stars at $40 \AA \mathrm{mm}^{-1}$. No systematic errors exceeding accidental errors of measurement were found. Personal and flexure errors were under examination. The scarcity of standard velocity stars earlier than F2 and in declinations suited to Allegheny should be remedied.

Heard seeks faint standard velocity stars suitable for calibration of objective-prism observations. A total of 122 spectra at $12 \AA \mathrm{mm}^{-1}$ and nine at $15 \AA \mathrm{mm}^{-1}$ from Dunlap and Victoria cover 24 stars, 21 of which have at least four spectra each. Seventeen seem suitable for adoption as standards but seven spectra of each star are desirable. Fehrenbach has spectrograms of some. Heard and Fehrenbach will prepare a combined list.

$G$. de Vaucouleurs with his wife continues interference filter observations (as reported at $I A U$ Symp. No. 30) of clusters, H II regions, and galaxies. For recession velocities less than $1100 \mathrm{~km} \mathrm{sec}^{-1}$ results agree well with $21-\mathrm{cm}$ values, but, thereafter blending of galaxy absorption $\mathrm{H}$ and $\mathrm{K}$ with night-sky $\mathrm{H}$ and $\mathrm{K}$ leads to underestimation of optical radial velocities by up to $30 \%$. This has important practical implications for estimation of the Hubble constant.

Davis Philip based his conclusions on the relative accuracy of image-tube and conventional spectrographs on observations of faint A-stars at high galactic latitudes (Table 1).

Table 1

Spectrograph and Telescope

Mt. Wilson B (100-in.)

Westinghouse Fiber Optics (84-in. KPNO)

Carnegie Tube (84-in. KPNO)
Dispersion at
$\mathbf{H} \gamma\left(\AA \mathrm{mm}^{-1}\right)$

86

69

50
Limiting mag. (Exp. $1^{\mathrm{h}}$, width $0.2 \mathrm{~mm}$ )

11.5

14

15
P.E. $\left(\mathrm{km} \mathrm{sec}^{-1}\right)$

$\pm 3$

$\pm 15$

$\pm 9$

Vera Rubin used an image tube in observing galaxies from a list by $M . S$. Roberts for which optical and $21-\mathrm{cm}$ velocities differed appreciably. The new optical measures agree much better. Published optical velocities can be appreciably in error.

Thackeray described tests of the 2-prism Cassegrain spectrograph with slit rotated in the direction of atmospheric dispersion. Measures of faint standard velocity stars have been made. Lloyd Evans continues observation of Cepheid binaries (frequency 15\%). Orbital elements of the single-lined binary hot sub-dwarf HD 49798 have been determined.

Batten described Fletcher's effort to obtain maximum precision from the Victoria coudé spectrograph at $2.5 \AA \mathrm{mm}^{-1}$. A systematic difference of $0.1 \mathrm{~km} \mathrm{sec}^{-1}$ has been found between velocities obtained using iron-arc comparison and discharge tube, the former being, apparently, correct. Batten will communicate this result to Commission 14. 
Pedoussaut studies statistics of spectroscopic binary stars. Of 858 known, 260 have UBV measures. Distribution in the galactic plane is nearly uniform, but there is an asymmetry perpendicular to the plane. Additional photometry is needed to show if the effect is real.

Fehrenbach measures radial velocities in the Large Magellanic Cloud region. The programme covers 494 member stars between magnitudes 9 and 13.5, and 1890 galactic stars. UBV photometry and spectroscopy at $73 \AA \mathrm{mm}^{-1}$ are well advanced.

Hube recalled that at Hamburg Bonneau reported that stars of types B8 and B9 with GCRV entries of $d$ and $e$ quality showed an apparent systematic error. On Thackeray's initiative eleven such stars had been observed at Pretoria, Toronto and Victoria. Hube's measures of all the plates showed no significant differences between the observatories. The effect is not explained by measurement difficulties due to diffuse spectrum lines.

D. S. Evans described the arrangement of the pages in the revision of the GCRV, now complete to $17 \mathrm{~h}$ R.A. Many colleagues had helped in proof-reading typescript copies. He hoped to finish early in 1971 .

A letter from $A b t$ suggested that the Commission concern itself with minimizing instrumental and guiding errors, with the encouragement of more observational work, and with improvement of storage and retrieval of numerical values of known velocities. This last project would be closely connected with any astronomical data center that may be created. 